\title{
A rating-based network selection game in heterogeneous systems
}

\author{
Vladimir Fux \\ Institut Telecom; Telecom Bretagne \\ 2, rue de la Chataigneraie CS 17607 \\ 35576 Cesson-Sevigne, FRANCE \\ e-mail: vladimir.fux@telecom-bretagne.eu
}

\author{
Patrick Maillé \\ Institut Telecom; Telecom Bretagne \\ 2, rue de la Chataigneraie CS 17607 \\ 35576 Cesson-Sevigne, FRANCE \\ e-mail: patrick.maille@telecom-bretagne.eu
}

\begin{abstract}
During the last years the problem of network selection in wireless heterogeneous systems has attracted a lot of attention. Expecting that next generation mobile devices will allow connections to different types of networks, it is interesting to investigate the outcome of selfish behavior in that context. It may then be necessary to introduce some mechanisms to drive users choices to some desirable directions - quality of service (QoS) optimization, energy consumption minimization, network revenue maximization -. In this paper, we define and investigate a system where users decide which network to connect to based on some ratings of networks, possibly computed from feedback sent by other users.

We then apply that model to investigate the pricing decisions made by network owners, in two different settings: a competition among several revenue-oriented operators, or a revenuemaximizing monopoly. The outcomes of those settings are compared, in terms of network usage and energy consumption.

Index Terms-Network selection; game theory; rating game; congestion control; quality of service;
\end{abstract}

\section{INTRODUCTION}

The term "Always Best Connected" was first coined by Gustafsson and Jonsson [4], who describe a system where users have several devices with different types of supported networks, and the system tries to keep the user connected to the internet at every moment of time. The authors provide a detailed business model and consider technical solutions required for the implementation of such a system. One of the questions raised in [4] was how to determine the most suitable network for a user in its current situation.

A network selection mechanism taking into account multiple factors, including the available throughput, security, cost, timeliness (which consists of delay, response time, and jitter) and reliability (which consists of burst error, average number of retransmissions per packet, and packet loss ratio) is proposed in [9]. That approach is mainly user-centric, and does not take into account provider utilities or negative externalities (congestion effects) among user choices.

Another approach is to consider both providers' and users' gain. [5] considers the network selection problem with respect to the preferences of both users and providers. The objective function considered is the weighted sum of two components - one represents the user satisfaction level, as the difference between their received rate with comparison to their required one, and the other shows the benefit of the access point, measured by users willingness to pay for their received rate. Then a greedy algorithm is proposed to optimize the global performance of the network. The decision is then centralized, whereas we consider a decentralized approach in this work, the decision of the network to join being left to the user.

In [2], users choose their network based on the expected delay, with the help of network probing. In that work, several strategies of delay information updating and algorithms for selecting a suitable network are considered, including decentralized approaches for collecting information about network congestions. In the model we develop, users also base their choice on some performance measure (in addition to the price) sensitive to congestion, that we interpret as a rating. The process of collecting and distributing those ratings is above the scope of this paper, but we could imagine that ratings are obtained from an aggregation of the feedbacks given by the users of the different networks.

In addition to proposing some Quality of Service (QoS) measure to base one's decision on, it is also interesting to investigate the performance of the system depending on user behavior. A classical framework to perform that task when users are selfish is that of noncooperative game theory [7]. In [3], a network selection game is played among users, while a resource allocation game is played, at a larger time scale, by providers. In those games, users select a provider and a frequency so as to minimize their perceived interference level, that depends on the number of users connected on the same frequency within some interfering distance, while at the higher game level providers select frequencies to operate in order to maximize the number of connected users. In [6], users select their network selfishly to minimize the price paid per successfully sent packet (that depends on congestion since all submitted packets are charged to their sender), while providers maximize revenue by playing on prices. That pricing game is shown to have a unique Nash equilibrium, where social welfare (the total value that is extracted by the system given the provider capacities) is maximized. In this paper, we also consider some leader-follower situations, where the leader(s) fix the prices on each network, anticipating the reaction of users (the followers). However, we do not only focus on provider revenues and economic efficiency, but also on the energy efficiency of the resulting prices. In addition, we 
consider two situations of the price setters: competition among revenue-oriented operators, and global revenue maximization by a single entity owning all access points.

The remainder of this paper is organized as follows. Section II presents the setting of our model, namely the network topology considered, the stakeholders and their strategic decisions, and specifies the hierarchy in the decisions taken. We establish some analytical results for the game played among users for fixed network prices in Section III. Then two possible settings for the pricing decisions are described (competition among providers, or collusion/monopoly) in Section IV, and the consequences in terms of global performance are analyzed in Section V. Some conclusions and directions for future work are given in Section VI.

\section{MODEL}

\section{A. Network topology}

In this paper we consider a system consisting of two networks. For the sake of simplicity, we assume that both networks have the same coverage area, as illustrated in Figure 1. Moreover, we consider that all mobile users own the technology allowing them to connect to both networks.

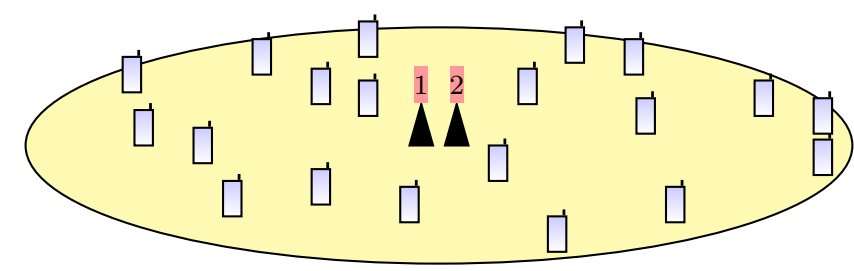

Fig. 1. Network topology considered in this paper.

\section{B. User behavior}

Users are sensitive to the QoS they experience, and to the price they are charged for the service. While the latter is clearly advertised by the networks, the former is less obvious to determine, since QoS estimations based on probing often involve some nonnegligible amount of uncertainty, due to the rapid changes in radio conditions. To cope with that problem, we consider a controller that computes in real time an averaged (over all users) value of the QoS level of each network, and propagates those levels to all users in the system. That average value will be called the rating of the considered network: it can be computed based on some feedback of the experienced QoS from all users (hence the averaging), or directly calculated by the access point based on the number of connected users. The details of that aspect are beyond the scope of this paper: we focus here on the dynamics implied by the rating scheme, and will consider that this rating depends on the level of congestion of each network (i.e., the number of connected users).

We consider that time is slotted; at each time slot users that are present in the system make a choice. Recall that the final decision is left to the user herself (instead of an algorithm implemented within the mobile terminal). To describe user behavior, we use the well-known logit model [8], where each user chooses a network based on its quality and price, but also on other individual criteria that we model as random variables (see [8] for details). In the case of two networks, the probability that a user $j$ chooses network $i \in\{1,2\}$ then equals, at each time slot:

$$
p_{i}^{j}=\frac{e^{\left(V_{i}^{j}-s^{j} P_{i}\right)}}{e^{\left(V_{1}^{j}-s^{j} P_{1}\right)}+e^{\left(V_{2}^{j}-s^{j} P_{2}\right)}},
$$

where $V_{i}^{j}$ is the current quality of network $i$ for user $j, P_{i}$ is the price per time slot of network $j$, and $s^{j}$ is the price sensitivity of user $j$ (that will be assumed to follow a given distribution over the user population).

1) Perceived quality and loyalty effect: At each time slot, the middle controller gathers information about the QoS experienced by users, and updates the network ratings. We chose the following update mechanism for the rating $Q_{i}^{t}$ at time $t$ :

$$
Q_{i}^{t}=\beta \cdot Q_{i}^{t-1}+(1-\beta) \bar{Q}_{i}{ }^{t-1},
$$

where $Q_{i}^{t-1}$ is the rating of network $i$ on period $t-1, \bar{Q}_{i}{ }^{t-1}$ is the (estimated) QoS computed by the central controller at period $t-1$, and $\beta \in(0,1)$ is a memory coefficient, that prevents ratings from changing too fast after a temporary QoS variation. It is easy to see that a bigger $\beta$ reduces the oscillations in $Q_{i}$, but in the other hand the information about the network congestion state then becomes less representative of the current situation.

The quality value $V_{i}^{j}$ in (1) can be considered as a simple rating (this quality value being then the same for all users), or alternatively we could consider this value to vary from user to user. More precisely, we will consider in this paper that $V_{i}^{j}$ contains a QoS-related term $Q_{i}$, that is modulated by the network (if any) that the user was attached to in the previous time slot. This way, we are modeling some loyalty effect, meaning that a user is reluctant to switch networks once he is connected to one. More precisely, we consider that the quality of network $i$ considered in (1) by user $j$ is $V_{i}^{j}=Q_{i}(1+\alpha)$ if user $j$ was with network $i$ during the previous time slot, and $V_{i}^{j}=Q_{i}$ otherwise. The parameter $\alpha>0$ can be interpreted as the loyalty value (or some cost corresponding to switching networks) of users. It introduces a bias in (1), that favors the decision to stay with the same network. Note that in this paper, we assume that all users have the same loyalty value $\alpha$.

The intuition about this system is that it should be selfregulating, i.e., independently of the QoS function used (delay, interference level, available bandwidth, ...), users should end up being distributed over the networks, in accordance with the quality and price levels. In other words, a situation where most users constantly choose the cheapest network is not possible in our system, because the rating of the congested network will degrade significantly, and consequently less users will choose that network in the next round.

2) User arrival and departure processes: We consider that at each time slot, the number of new users entering the game (i.e., willing to benefit from the service) is randomly 
distributed, following a Poisson distribution with mean value $\lambda$.

Users leave the system after some (randomly distributed) time. We assume that this service duration follows a memoryless distribution, i.e. at each time slot there is a probability $1-q$ that the user ends its service (call) at the end of the slot, independently for each user participating in the system. It is easy to see that the expected number of users in the system then converges to $\frac{\lambda}{1-q}$.

\section{Provider utilities}

For some settings considered in this paper, the providers ${ }^{1}$ will be considered as players, who decide the price to charge each network. Since $P_{i}$ is a price per time slot, the average revenue of network $i$ per time slot is simply

$$
R_{i}=P_{i} n_{i}
$$

where $n_{i}$ is the expected (average) number of users connected to network $i$. In this paper, when prices are set by providers, we will assume that the objective is to maximize that revenue, i.e., we ignore any other management or energy costs borne by providers. Notice that because of the choice among networks described in (1), the revenue of each network depends on the price profile $\left(P_{1}, P_{2}\right)$. In addition, the average number $\lambda$ of new entrants introduced in the previous subsection can reasonably be considered to depend on the prices set by networks.

As a result, in the competitive situation, we are faced with a noncooperative game played by providers to maximize revenue.

\section{Decision hierarchy: a two-stage game}

We consider that prices are fixed for a long time period (with respect to the average user service duration), so that they can be considered as fixed when studying the process of users selecting networks. Also, we assume that the pricesetters (the providers) are able to anticipate the behavior of the users. In that sense, we have a multiple-leader multiplefollower game, that we analyze using backward induction: the pricing decisions are made, using the knowledge of the corresponding outcome for the user interactions.

\section{ANALYTICAL RESUlTS FOR FIXED NETWORK PRICES}

In this section, we analyze the lower level of the game, that is the one played among users, selecting their network based on prices and quality. We therefore assume in this section that the prices $P_{1}$ and $P_{2}$ are fixed and constant. In that context, we derive some analytical results regarding the steady-state situation of the stochastic process defined in Section II.

Note that for this analytical part, we make the simplifying assumption that user price sensitivities are re-drawn from the same distribution at each time slot. This is of course incorrect in reality, since we interpret price sensitivities as user-specific parameters: in our simulations we actually keep

\footnotetext{
${ }^{1}$ We will indifferently use the terms "provider" and "operator", to refer to the entities controlling the networks.
}

that sensitivity constant for the whole duration of a user's connection. Note however that in the case without any loyalty effect, that assumption does not lead to a bias in the expected number of users in each network, but only in the number of handovers (which is why the latter metric is only evaluated through simulations).

\section{A. Existence of a stationary distribution}

We first remark that the discrete-time process $\left(n_{1}^{t}, n_{2}^{t}\right)$, giving the evolution of the number of users connected to each network, is a Markov chain. Indeed, at each time slot the quality of service $Q_{i}^{j}$ considered by users for their next decision, and the number of new arrivals, only depend on the current state (and not on the previous ones). Since those values are the only ones determining the distribution of $\left(n_{1}^{t+1}, n_{2}^{t+1}\right)$, the process satisfies the Markov property. It is easy to check that this Markov chain is irreducible and aperiodic: just consider that any transition $\left(n_{1}, n_{2}\right) \rightarrow(0,0)$ has a non-zero probability, as well as any transition $(0,0) \rightarrow\left(n_{1}, n_{2}\right)$.

To establish that the Markov chain is ergodic (and thus, admits a stationary distribution), it remains to show that at least one state is positive recurrent. This can be done easily by considering the state $(0,0)$, which allows us to reason only on the total number of users regardless of their network choice. The total number of users in the system is itself a (discrete-time) Markov process, that is irreducible and aperiodic, and obviously positive recurrent since the number of users converges to the finite value $\lambda /(1-q)$, as pointed out in Subsection II-B2. Therefore all its states are recurrent, including the state with no users that coincides with the state $(0,0)$ of the process $\left(n_{1}^{t}, n_{2}^{t}\right)$.

Consequently, the process $\left(n_{1}^{t}, n_{2}^{t}\right)$ is an ergodic Markov chain, that therefore admits a stationary distribution: after some time, the probabilities of visiting each state $\left(n_{1}, n_{2}\right)$ do not change. In particular, we can then claim that the number of users in each network has a mathematical expectation, around which it will oscillate during a process trajectory.

\section{B. Expected number of users in each network}

For simplicity reasons, we first consider the case without loyalty effect (i.e., $\alpha=0$ ), so that the perceived network ratings are the same for all users, i.e., $V_{i}^{j}=Q_{i}$. We assume the price sensitivities of users to be uniformly distributed on the interval $[a, b]$, for $0 \leq a<b$. Thus, when the quality scores $\left(Q_{1}, Q_{2}\right)$ of the previous time slot and the number of users $n^{t}$ in the network are given, the mathematical expectation of the number of users choosing network $i$ at time slot $t$ is, with $\bar{i}:=\{1,2\} \backslash\{i\}$ :

$$
\begin{aligned}
\mathbb{E}\left[n_{i}^{t}\right] & =\sum_{j=1}^{n^{t}} \mathbb{E}_{s}\left[\mathbb{E}\left[\mathbb{1}_{\{\text {user } j \text { selects network } i\}} \mid s_{j}=s\right]\right] \\
& =n^{t} \int_{a}^{b} \frac{e^{\left(Q_{i}-x P_{i}\right)}}{e^{Q_{1}-x P_{1}}+e^{Q_{2}-x P_{2}}} \frac{1}{(b-a)} d x \\
& =n^{t}\left[1+\frac{1}{\left(P_{i}-P_{i}\right)(b-a)} \ln \frac{\left.1+e^{Q_{\bar{i}}-Q_{i}} e^{-b\left(P_{\bar{i}}-P_{i}\right.}\right)}{1+e^{Q_{\bar{i}}-Q_{i}} e^{-a\left(P_{\bar{i}}-P_{i}\right)}}\right]
\end{aligned}
$$


when $P_{1} \neq P_{2}$. If $P_{1}=P_{2}$, then

$$
E\left[n_{i}^{t}\right]=\frac{n^{t}}{1+e^{Q_{\bar{i}}-Q_{i}}} .
$$

When there is some loyalty effect (i.e., $\alpha>0$ ), the computation is a bit more complicated since the perceived rating is user-specific: we have $V_{i}^{j}=Q_{i}\left(1+\alpha \mathbb{1}_{\left\{j \in N_{i}^{t-1}\right\}}\right)$, where $N_{i}^{t}$ represents the set of users connected to network $i$ during time slot $t$, and $N^{t}:=N_{1}^{t} \cup N_{2}^{t}$. We then have for a user present at time slot $t$,

$$
\begin{aligned}
& j \in N_{i}^{t-1} \Rightarrow p_{i}^{j}(t)=\frac{e^{\left(Q_{i}(1+\alpha)-s^{j} P_{i}\right)}}{e^{\left(Q_{i}(1+\alpha)-s^{j} P_{i}\right)}+e^{\left(Q_{\bar{i}}-s^{j} P_{\bar{i}}\right)}} \\
& j \in N_{\bar{i}}^{t-1} \Rightarrow p_{i}^{j}(t)=\frac{e^{\left(Q_{i}-s^{j} P_{i}\right)}}{e^{\left(Q_{i}-s^{j} P_{i}\right)}+e^{\left(Q_{\bar{i}}(1+\alpha)-s^{j} P_{\bar{i}}\right)}} \\
& j \notin N^{t-1} \Rightarrow p_{i}^{j}(t)=\frac{e^{\left(Q_{i}-s^{j} P_{i}\right)}}{e^{\left(Q_{i}-s^{j} P_{i}\right)}+e^{\left(Q_{\bar{i}}-s^{j} P_{\bar{i}}\right)}} .
\end{aligned}
$$

Consequently, we have, if we define $m_{i}:=\left|N_{i}^{t-1} \cap N_{i}^{t}\right|$,

$$
\begin{aligned}
\mathbb{E}\left[n_{i}^{t}\right]= & m_{i} \mathbb{E}\left[\frac{e^{\left(Q_{i}(1+\alpha)-s^{j} P_{i}\right)}}{e^{\left(Q_{i}(1+\alpha)-s^{j} P_{i}\right)}+e^{\left(Q_{\bar{i}}-s^{j} P_{\bar{i}}\right)}}\right]+ \\
& m_{\bar{i}} \mathbb{E}\left[\frac{e^{\left(Q_{i}-s^{j} P_{i}\right)}}{e^{\left(Q_{i}-s^{j} P_{i}\right)}+e^{\left(Q_{\bar{i}}(1+\alpha)-s^{j} P_{\bar{i}}\right)}}\right]+ \\
& \left(n^{t}-m_{1}-m_{2}\right) \mathbb{E}\left[\frac{e^{\left(Q_{i}-s^{j} P_{i}\right)}}{e^{\left(Q_{i}-s^{j} P_{i}\right)}+e^{\left(Q_{\bar{i}}-s^{j} P_{\bar{i}}\right)}}\right],
\end{aligned}
$$

where the three summands respectively represent the expected number of users which were in network $i$ and did not change their choice, the expected number of users which migrated from network $\bar{i}$ to network $i$, and the expected number of newly arrived users that chose network $i$.

After some algebra, we obtain, conditionally on $n^{t-1}, m_{1}$ and $m_{2}$, and on the values of $Q_{1}$ and $Q_{2}$ at the previous time slot,

$$
\begin{aligned}
\mathbb{E}\left[n_{i}^{t}\right] & =m_{i}\left[1+K_{i} \ln \frac{1+e^{Q_{\bar{i}}-Q_{i}(1+\alpha)} e^{-b\left(P_{\bar{i}}-P_{i}\right)}}{1+e^{Q_{\bar{i}}-Q_{i}(1+\alpha)} e^{-a\left(P_{\bar{i}}-P_{i}\right)}}\right] \\
& +m_{\bar{i}}\left[1+K_{i} \ln \frac{\left.1+e^{Q_{\bar{i}}-Q_{i}} e^{-b\left(P_{\bar{i}}-P_{i}\right.}\right)}{1+e^{Q_{\bar{i}}(1+\alpha)-Q_{i}} e^{-a\left(P_{\bar{i}}-P_{i}\right)}}\right] \\
& +\left(n^{t}-m_{1}-m_{2}\right)\left[1+K_{i} \ln \frac{\left.1+e^{Q_{\bar{i}}-Q_{i}} e^{-b\left(P_{\bar{i}}-P_{i}\right.}\right)}{1+e^{Q_{\bar{i}}-Q_{i}} e^{-a\left(P_{\bar{i}}-P_{i}\right)}}\right],
\end{aligned}
$$

for the case when $P_{1} \neq P_{2}$, with $K_{i}:=\frac{1}{\left(P_{\bar{i}}-P_{i}\right)(b-a)}$. When prices are equal, we have

$\mathbb{E}\left[n_{i}^{t}\right]=\frac{m_{i}}{1+e^{Q_{\bar{i}}-Q_{i}(1+\alpha)}}+\frac{m_{\bar{i}}}{1+e^{Q_{\bar{i}}(1+\alpha)-Q_{i}}}+\frac{n^{t}-m_{i}-m_{\bar{i}}}{1+e^{Q_{\bar{i}}-Q_{i}}}$.

Finally, to have results conditionally on the user repartition at time slot $t-1$ only, we can plug in the previous expression the relations $\mathbb{E}\left[m_{i}\right]=n_{i}^{t-1} q$ and $\mathbb{E}\left[n^{t}\right]=q n^{t-1}+\lambda$, where we recall that $q$ is the probability that a user continues his service at the next time slot and $\lambda$ is the expected number of new entrants at each time slot.

\section{Average churn rate}

In this subsection, we focus on the phenomenon of churn, that is, the fact that users switch networks during their communication. This can be due to the mobility of users (that is not considered here), to some temporary changes in the network conditions (reflected by a change in the quality values $\left(Q_{i}\right)$ ), or to some user-specific criteria.

Quantifying the occurrence of that phenomenon is of crucial importance to the network management, since switching networks incurs energy-costly procedures to perform the handover. The frequency of churns is therefore directly linked to the overall energy consumption of the global network.

Using the same method as before, the expectation of the number of network changes $h^{t}$ at time slot $t$ (conditionally on the situation at time slot $t$ ) can be computed:

$$
\begin{aligned}
\mathbb{E}\left[h^{t}\right]= & n_{1}^{t-1} q\left[1-K_{1} \ln \frac{1+e^{Q_{1}(1+\alpha)-Q_{2}} e^{-b\left(P_{1}-P_{2}\right)}}{1+e^{Q_{1}(1+\alpha)-Q_{2}} e^{-a\left(P_{1}-P_{2}\right)}}\right] \\
& +n_{2}^{t-1} q\left[1+K_{1} \ln \frac{\left.1+e^{Q_{2}(1+\alpha)-Q_{1}} e^{-b\left(P_{2}-P_{1}\right.}\right)}{1+e^{Q_{2}(1+\alpha)-Q_{1}} e^{-a\left(P_{2}-P_{1}\right)}}\right]
\end{aligned}
$$

still with $K_{1}=\frac{1}{\left(P_{2}-P_{1}\right)(b-a)}$.

\section{Illustrations}

In this subsection, we present some simulations that illustrate the selection game we have defined, and the analytical results of this section. Two cases are considered: one without loyalty effect $(\alpha=0)$, and one with a loyalty value $\alpha=3$. Unless specified otherwise, the parameters used in the simulations are the following:

- range of the price sensitivity values $s^{j}:[a, b]=[0,0.4]$,

- average number of new entrants per time unit: $\lambda=200$,

- probability of leaving the system at the end of the current time slot: $1-q=0.2$,

- quality score of network $i$ of the form ${ }^{2} \bar{Q}_{i}=1-$ $\left(n_{i}^{t} / C_{i}\right)^{2}$, with $C_{i}$ the capacity of network $i$,

- networks of respective capacities $C_{1}=1000, C_{2}=600$,

- respective prices of each network $P_{1}=9, P_{2}=8$,

- memory effect in the computation of $Q_{i}$ in (2): $\beta=0.9$.

Figure 2 shows the evolution of the number of users in each network, without any loyalty effect. We remark that due to the inner probabilistic nature of user choices, those numbers do not converge to a given value. However, after a few iterations the system is close to its steady state, and the number of users in each network oscillates around their expected value. Note here that the expectation on each iteration is computed from (4)-(5), but using previous iteration's expectations $\mathbb{E}\left[n_{i}^{t-1}\right]$ instead of the real values $n_{i}^{t-1}$. Therefore, the curves for $\mathbb{E}\left[n_{1}^{t}\right]$ and $\mathbb{E}\left[n_{2}^{t}\right]$ are completely deterministic. We observe that those expected values are very good estimators of the average values of $n_{1}^{t}$ and $n_{2}^{t}$, respectively.

\footnotetext{
${ }^{2}$ Note that we could also consider totally different forms for $Q_{1}\left(n_{1}\right)$ and $Q_{2}\left(n_{2}\right)$, that could reflect the different technologies used in the heterogeneous network. With the form taken here, the only heterogeneity lies in the capacity differences among networks.
} 


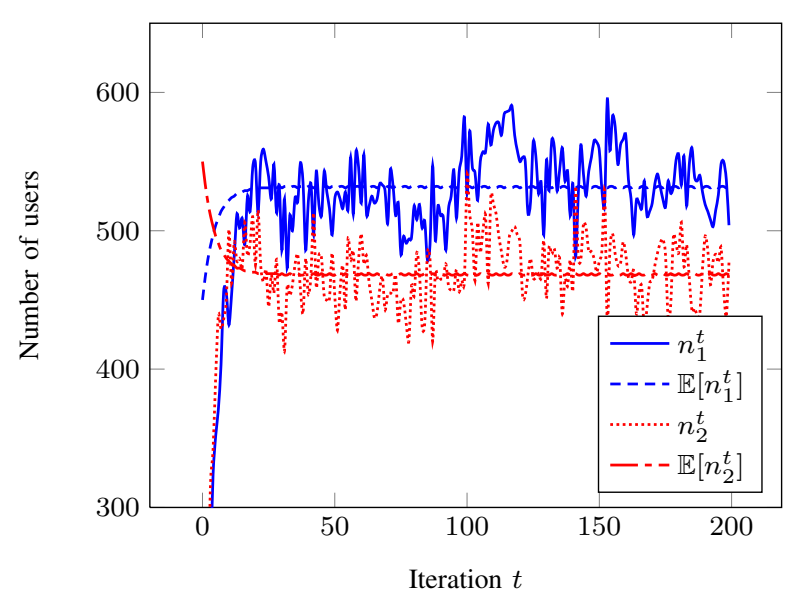

Fig. 2. Number of users in each network, without loyalty effect.

We plot in Figure 3 the corresponding values of the ratings $\left(Q_{1}, Q_{2}\right)$, computed over time following (2). Similarly to the number of users in each network, after the starting phase where ratings are high due to the small number of users, ratings stabilize around a constant value, still with oscillations. Note however that the amplitude of the oscillations are smaller than for the number of users, due to the memory effect introduced in (2) that smoothes the variations.

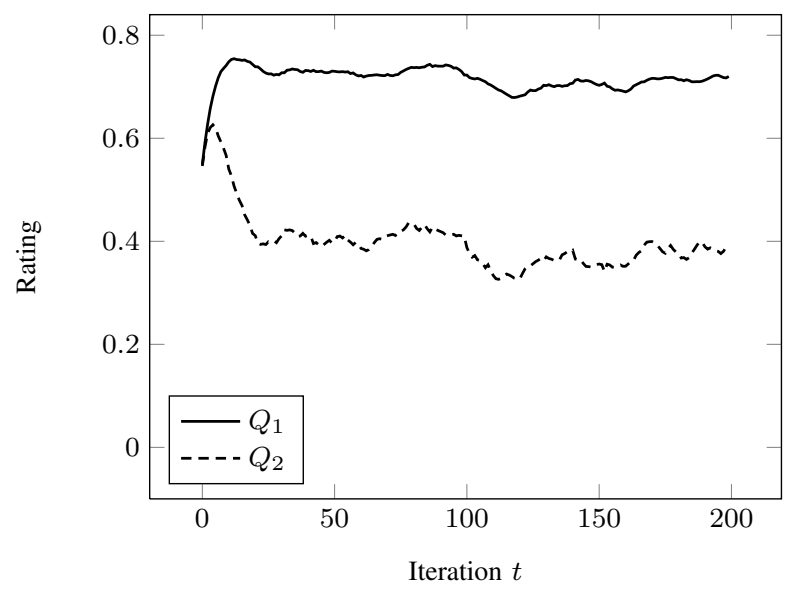

Fig. 3. Rating dynamics, without loyalty effect.

Figures 4 and 5 are the counterparts of Figures 2 and 3, but with a loyalty value $\alpha=3$. We remark as expected that oscillations still take place, but to a smaller extent with respect to the no-loyalty case. Notice also that the loyalty phenomenon affects not only the number of handovers (the churn effect), but also the average balance between networks: users tend to go more to network 1 when the loyalty effect is introduced. The explanation of this is as follows: without the loyalty effect the majority of users already used to prefer the first network, and the loyalty effect then retains them from changing networks. Users spend less time "exploring" the other network, and prefer to stick to their current one (in most cases, their preferred one). Another direct consequence is that the loyalty effect tends to reduce the difference in the steadystate ratings $Q_{1}$ and $Q_{2}$ : users mostly preferring network 1 and churning less, that network becomes more congested, hence a reduction in its rating.

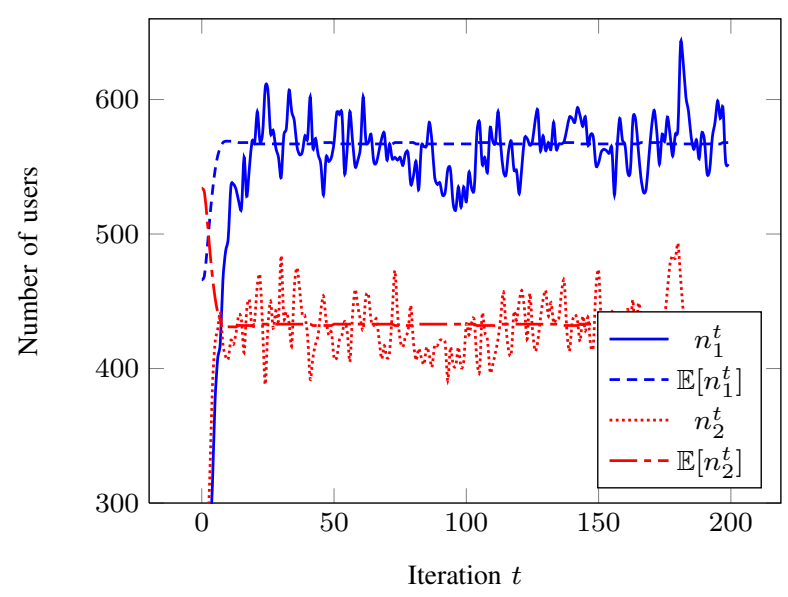

Fig. 4. Number of users in each network, with loyalty effect.

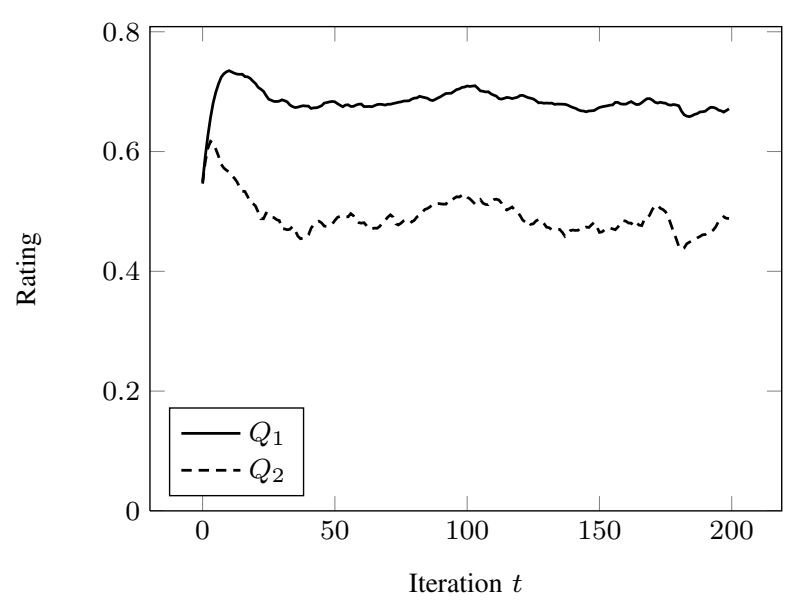

Fig. 5. Rating dynamics, with loyalty effect.

Finally, Figure 6 illustrates the dependence of the loyalty coefficient on the churn phenomenon: as expected, a larger reluctance to switch networks reduces churn significantly, even if the other network is temporarily more attractive.

\section{E. Computing the steady-state user distribution}

The simulation results of Subsection III-D suggest that the mathematical expectations of the number of users (computed by recursively estimating the number of users at each time slot) are very close to the steady-state average values. This is partially due to the memory effect $\beta$ : when $\beta$ tends to 1 then the quality values $Q_{i}$ converge to a fixed value. Considering that limit case when $\beta \rightarrow 1$, we expect that without loyalty effect, the average number $n_{i}^{*}$ of users in network $i$ is close 


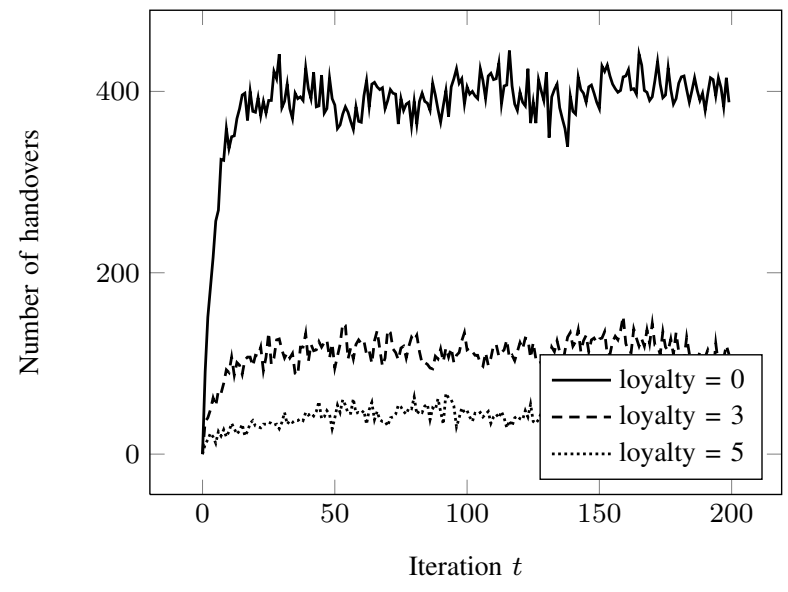

Fig. 6. Number of handovers per time slot.

to the solution of the following fixed-point equation:

$$
n_{i}^{*}=\frac{\lambda}{1-q}\left[1+K_{i} \ln \frac{\left.1+e^{Q_{\bar{i}}\left(n_{\bar{i}}^{*}\right)-Q_{i}\left(n_{i}^{*}\right)} e^{-b\left(P_{\bar{i}}-P_{i}\right.}\right)}{1+e^{Q_{\bar{i}}\left(n_{\bar{i}}^{*}\right)-Q_{i}\left(n_{i}^{*}\right)} e^{-a\left(P_{\bar{i}}-P_{i}\right)}}\right],
$$

with $K_{i}=\frac{1}{\left(P_{i}-P_{i}\right)(b-a)}$

With some loyalty effect, that fixed-point equation becomes:

$$
\begin{aligned}
n_{i}^{*} & =n_{i}^{*} q\left[1+K_{i} \ln \frac{1+e^{Q_{\bar{i}}\left(n_{\bar{i}}^{*}\right)-Q_{i}\left(n_{i}^{*}\right)(1+\alpha)} e^{-b\left(P_{\bar{i}}-P_{i}\right)}}{1+e^{Q_{\bar{i}}\left(n_{\bar{i}}^{*}\right)-Q_{i}\left(n_{i}^{*}\right)(1+\alpha)} e^{-a\left(P_{\bar{i}}-P_{i}\right)}}\right] \\
& +\left(\frac{\lambda}{1-q}-n_{i}^{*}\right) q\left[1+K_{i} \ln \frac{1+e^{Q_{\bar{i}}\left(n_{\bar{i}}^{*}\right)(1+\alpha)-Q_{i}\left(n_{i}^{*}\right)} e^{-b\left(P_{\bar{i}}-P_{i}\right)}}{1+e^{Q_{\bar{i}}\left(n_{\bar{i}}^{*}\right)(1+\alpha)-Q_{i}\left(n_{i}^{*}\right)} e^{-a\left(P_{\bar{i}}-P_{i}\right)}}\right] \\
& +\lambda\left[1+K_{i} \ln \frac{\left.1+e^{Q_{\bar{i}}\left(n_{\bar{i}}^{*}\right)-Q_{i}\left(n_{i}^{*}\right)} e^{-b\left(P_{\bar{i}}-P_{i}\right.}\right)}{1+e^{Q_{\bar{i}}\left(n_{\bar{i}}^{*}\right)-Q_{i}\left(n_{i}^{*}\right)} e^{-a\left(P_{\bar{i}}-P_{i}\right)}}\right] .
\end{aligned}
$$

Remark that Equations (6) and (7) can be solved numerically.

\section{FIXING NETWORK PRICES: NUMERICAL ANALYSIS IN DIFFERENT SETTINGS}

We now consider the higher-level of our game, that consists in provider(s) setting the prices $P_{1}$ and $P_{2}$. Since Equations (6) and (7) give a relation between the price profile $\left(P_{1}, P_{2}\right)$ and the average number of users on each network, it is possible to investigate how providers act in order to maximize their revenue.

First of all, we introduce an elastic (i.e., price-sensitive) demand, that prevents providers from charging the maximum possible price to maximize revenue. We assume here that the average number of user arrivals per time period depends on providers' prices as follows:

$$
\lambda\left(P_{1}, P_{2}\right)=\lambda_{\max }\left(1-\frac{P_{1}+P_{2}}{P}\right),
$$

where $P$ represents a price above which no one wants to use the network services, and $\lambda_{\max }$ is the number of users that would use the system if services were free. Note that the demand in (8) can be derived from classical linear demand functions, often used in the literature [1]: there could be two potential sources of demand, of the form $\lambda_{1}=A-\eta P_{1}+\gamma P_{2}$ and $\lambda_{2}=B-\eta P_{2}+\gamma P_{1}$, where $\eta$ (resp. $\gamma$ ) represent the direct (resp. indirect) effect of the price of an operator (resp., its competitor). Aggregating those demands, to consider that users enter the game based on those and then select a network, we obtain the form given in (8).

We now investigate how the prices $P_{1}$ and $P_{2}$ are fixed, depending on the relation between the network owners.

\section{A. The noncooperative case: price competition}

We first consider the situation where both networks are controlled by different entities (operators), that do not collaborate. The operators then play a pricing game to attract customers, but still making revenue. Their strategic choice is then driven by the maximization of their payoff, that is their revenue as expressed in (3).

The analysis of the two-player noncooperative game is then performed numerically: we look for a Nash equilibrium [7] $\left(P_{1}^{*}, P_{2}^{*}\right)$ as a price profile such that $P_{i}^{*}$ is the best that operator $i$ can play when its competitor sets $P_{\bar{i}}^{*}$ so as to maximize its revenue. The Nash equilibrium is indeed a situation from which no player can improve its own payoff through a unilateral strategic change, i.e., an intersection point (if any), of the best-response curves of the two operators.

Figure 7 plots the best-response prices of both operators, for the parameter values given in Subsection III-D with loyalty effect, except that we consider the elastic demand case with $\lambda_{\max }=200$ and $P=20$, and we take price sensitivity values distributed over the interval $[a, b]=[0,0.5]$. We observe that

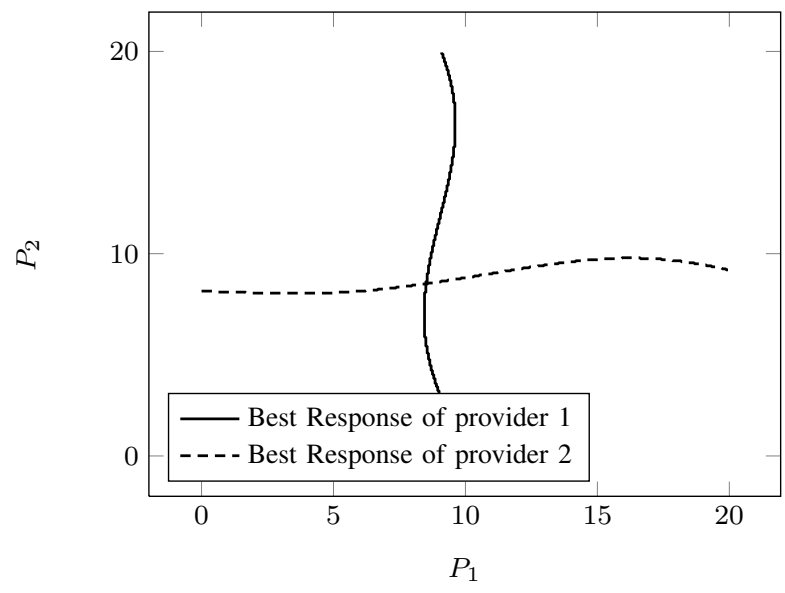

Fig. 7. Best-response prices.

the game has a unique Nash equilibrium, an observation we also made for the other parameter values tried. Interestingly, remark in Figure 7 that best-response prices are not necessarily monotonous in the price of the competitor.

\section{B. The cooperative case: a monopoly situation}

We will also consider the situation where both networks are owned by the same entity (that then acts as a monopolist) fixing prices to maximize the global revenue $P_{1} n_{1}^{*}+P_{2} n_{2}^{*}$.

Equivalently, the same outcome is reached when two operators control one network each, but decide to collude and set prices to maximize the sum of their revenues, possibly 
through some agreements regarding the sharing of the benefits of collusion.

\section{Global PERFormance OF THE DIFFERENT SETTINGS}

In this section, we compare the competitive and cooperative (monopoly) situations, in terms of different performance criteria. The parameters taken for the numerical results shown here are those of Subsection III-D.When the ratio $C_{2} / C_{1}$ varies, we actually fix $C_{1}$ to 1000 , and have $C_{2}$ vary from 100 to 1000 .

\section{A. Network prices}

With a loyalty coefficient $\alpha$ varying from 0 to 6 , we did not find any significant changes in equilibrium prices for both the monopoly and competition case. Figure 8 plots the equilibrium prices depending on the heterogeneity of the network (expressed by the ratio $C_{2} / C_{1}$ ). Here we observe that when heterogeneity decreases (i.e., $C_{2} / C_{1}$ gets closer to 1 ), prices for both settings converge to different values, and price in the competition case is lower than with a monopoly. In the competition situation, both providers tend to monotonically decrease their prices when $C_{2}$ increases. The price decrease for network 1 is obvious, because if the competitor increases the quality of its product, it then has to decrease price. For the second provider it is different: with the rise of the capacity of its network, operator is interested in attracting more users in the system, which it does by decreasing its price.

On the other hand, a monopolist is interested in charging a small price for the services in the network with bad capacity, and a high price for the network with better capacity, because in this case, a larger number of clients is attracted to the system (because of the total demand (8), that depends on the average of both prices, hence the low $P_{2}$ ), and because of congestion many of them will choose the largest (least congestionsensitive) network, thus increasing the total revenue with a quite high $P_{1}$.

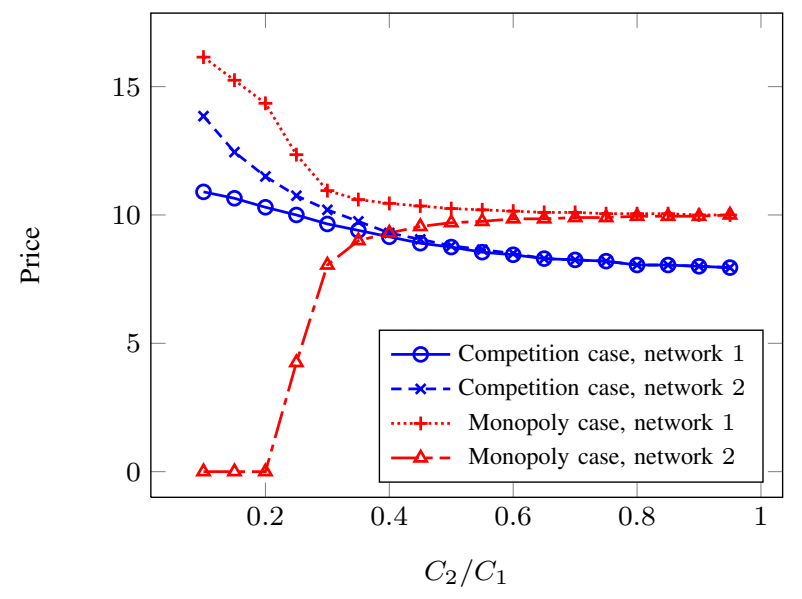

Fig. 8. Equilibrium prices in each network, versus capacity heterogeneity $C_{2} / C_{1}$.

\section{B. Number of users in each network}

Very small changes of in the number of users in each network were noticed when the loyalty coefficient varies, which is consistent with the results of the previous section. In the same vein, the total number of users in the competition case appears to be bigger than in the monopoly case.

Figure 9 highlights the influence of the network heterogeneity on the user repartition among networks. As expected, in all

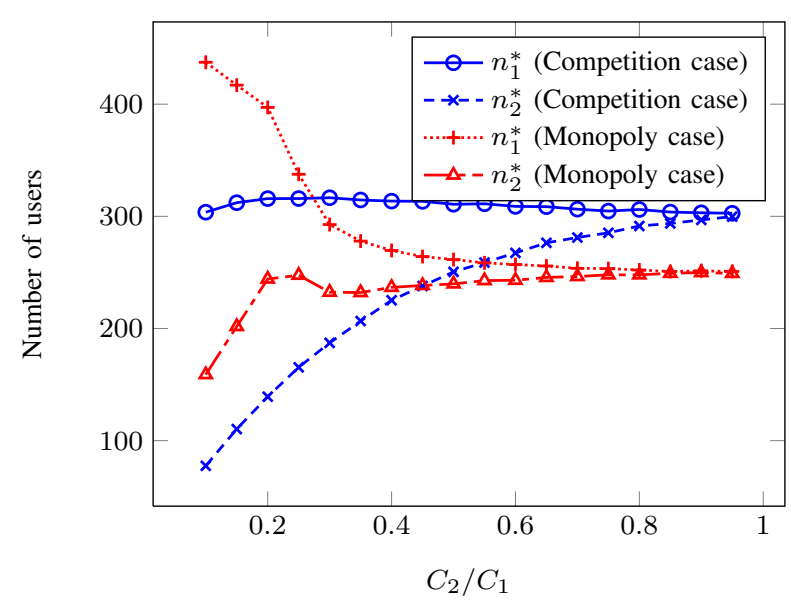

Fig. 9. Equilibrium number of users in each network, versus capacity heterogeneity $C_{2} / C_{1}$.

cases the largest network attracts more users. In accordance with Figure 8 , when $C_{2} / C_{1}$ is close to 1 , the total number of users in the competitive case is higher than in the monopoly case for each network.

\section{Distribution of user sensitivities to prices among networks}

It is interesting to see how a user's sensitivity to prices influences her network choices. This is illustrated in Figure 10, where the average sensitivity to price of users selecting each network is plotted.

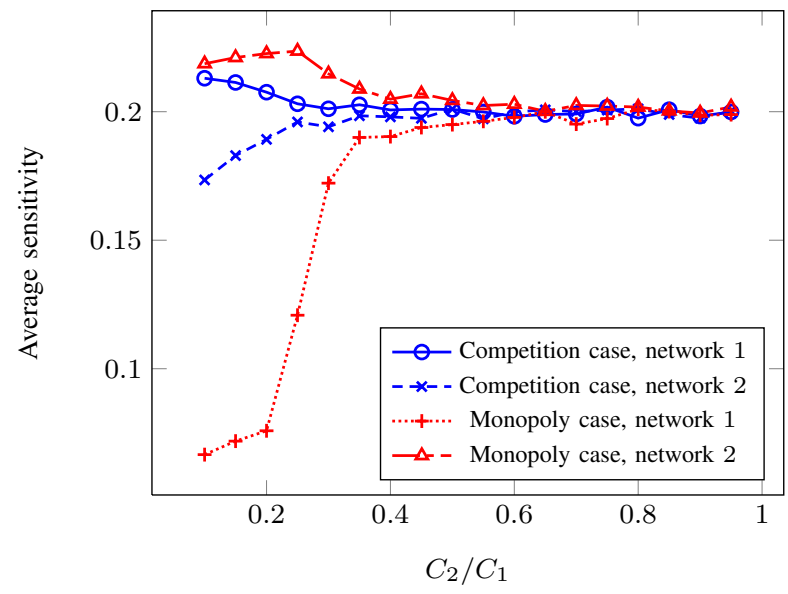

Fig. 10. Average user sensitivities of users in each network, versus capacity heterogeneity $C_{2} / C_{1}$. 
We observe that the monopoly leads to a strong discrimination of users according to their price sensitivity: when the system is very heterogeneous $\left(C_{2} / C_{1}\right.$ small), only users with a very low price sensitivity choose network 1 (that is the most expensive one but also the one with the best QoS). Note that the tendency is inverted for the competition case (network 1 tends to be chosen by less price-sensitive users than network 2 ), but the difference is much smaller. This can also be an argument in favor of the competition situation: the monopoly may lead to strong inequalities among users, where only "rich" users will benefit from a very good QoS.

\section{Energy consumption}

Finally, we focus on the energetic performance of the competitive versus monopolistic situations. Figure 11 displays the average user's energy consumption (AEC) dynamics depending on the loyalty effect parameter $\alpha$. We compute the

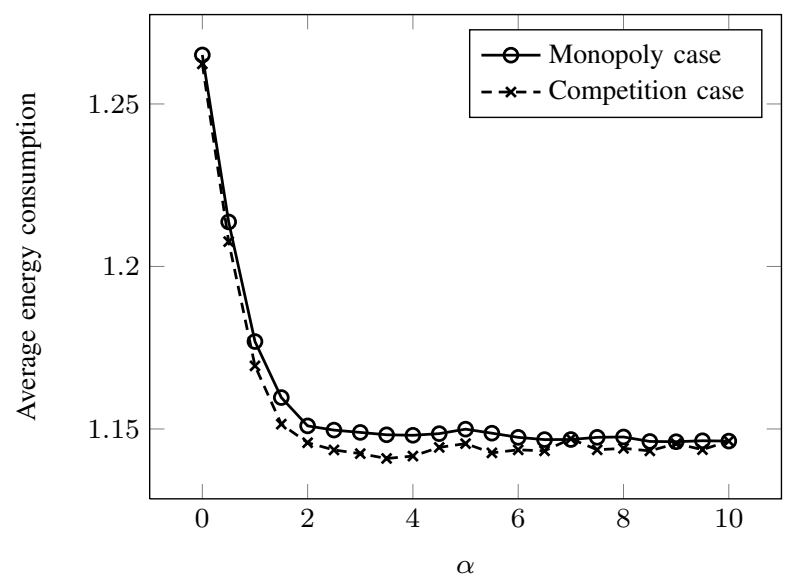

Fig. 11. Energy consumption for different cases

AEC as the average value of $e_{i(j)}+v \mathbb{1}_{\left\{j \in N_{\bar{i}(j)}^{t-1}\right\}}$, where $e_{i}$ is the energy that a user consumes per time slot when connected to network $i, i(j)$ is the network chosen by $j$ at the current time slot, and $v$ is the energy cost of a handover, that takes place if the user was attached to the other network at the previous time slot. The following values have been considered here: $e_{1}=1, e_{2}=1.3, \lambda_{\max }=200, P=20, q=0.2, v=0.3$.As expected, for both cases the AEC value decreases when the loyalty effect becomes more significant, mainly because of the decrease in the number of handovers. We also notice a slightly smaller energy consumption in the competitive case with respect to the monopolistic one, but with small differences (less than 1\%).

\section{CONCLUSION}

In this paper, we have introduced a model of network selection by wireless users in an heterogeneous network. In that systems, users make their choice based on networks' ratings, that are computed and distributed by a third-part entity, possibly using feedbacks from users' experienced QoS. We have investigated the model dynamics, and we proved that the numbers of users in networks oscillate around their expectation values, for which we have provided a good estimate through an analytical expression.

Those results can be used to forecast providers' revenues, the handover frequency, and the energy consumption. We have considered two cases of system management -by a single entity, or by competitive providers-, with revenue as the payoff and prices as the strategies in both cases. Those cases have been compared in terms of prices, network usage, and energy consumption, highlighting some interesting results like the fact that monopolistic situations are likely to lead to very unfair outcomes.

The model can be extended in several directions. It would first be interesting to consider different coverage areas for both networks, so that only some fraction of users would have a choice to make. Second, the mobility of users, moving from one area to another, would be worth considering. Finally, we intend to model not only two cells, but two cellular networks covering a wide area, with possibly different cell dimensions for each (representative of the different technologies considered).

\section{ACKNOWLEDGEMENTS}

This work has been partially funded by the French Bretagne Region through the ARED program, and by the Fondation Telecom through the "Futur\&Ruptures" program.

\section{REFERENCES}

[1] F. Bernstein and A. Federgruen, "A general equilibrium model for industries with price and service competition," Operations Research, vol. 52, no. 6, pp. 868-886, 2004

[2] B. Cassell, T. Aalperovich, M. Wellman, and B. Noble, "Access point selection under emerging wireless technologies," in Proc. of 6th workshop on the Economics of Networks, Systems, and Computation (NetEcon), San Jose, CA, USA, June 2011.

[3] M. Cesana, I. Malanchini, and A. Capone, "Modelling network selection and resource allocation in wireless access networks with non-cooperative games," in Proc. of 5th IEEE International Conference on Mobile Ad Hoc and Sensor Systems (MASS), Atlanta, GA, USA, September 2008, pp. 404-409.

[4] E. Gustafsson and A. Jonsson, "Always best connected," Wireless Communications, IEEE, vol. 10, no. 1, pp. 49-55, Feb 2003.

[5] P. Kosmides, A. Rouskas, and M. Anagnostou, "Network selection in heterogeneous wireless environments," in Proc. of 18th International Conference on Telecommunications (ICT), Ayia Napa, Cyprus, May 2011, pp. 250-255.

[6] P. Maillé and B. Tuffin, "Analysis of price competition in a slotted resource allocation game," in Proc. of IEEE INFOCOM, Phoenix, AZ, USA, April 2008, pp. 888-896.

[7] M. J. Osborne and A. Rubinstein, A Course in Game Theory. MIT Press, 1994.

[8] S. Patel and A. Schlijper, "Models of consumer behaviour," available online: http://www.maths-in-industry.org/miis/38/1/Consumers.pdf, 2010.

[9] Q. Song and A. Janalipour, "A network selection mechanism for next generation networks," in Proc. of IEEE International Conference on Communications (ICC), vol. 2, Seoul, South Korea, May 2005, pp. 14181422 . 